\title{
Controlled anisotropic wetting behaviour of multi-scale slippery surface structure of non fluoro polymer composite
}

\author{
B. N. Sahoo, B. Kandasubramanian* , B. Sabarish \\ Department of Materials Engineering, Defence Institute of Advanced Technology, Girinagar, 411025 Pune, India
}

Received 2 May 2013; accepted in revised form 22 July 2013

\begin{abstract}
A facile process for in-situ modification of surface properties of Waste Expanded Polystyrene (WEP)/graphite film produced by spin coating technique has been described. The additives undergo spontaneous surface agglomeration with formation of islands of forest of flake structure during the spinning process. This results in polymer films with enhanced roughness and highly hydrophobic surfaces. Wettability was analyzed using static water contact angle, surface morphology was observed using atomic force microscopy (AFM) and field emission scanning electron microscopy (FE-SEM). The polymer composite exhibited maximum water contact angle (WCA) of $129^{\circ}$. Surface texture reveals the variation of surface roughness which enables anisotropic surface wettability property. The present work exhibits promising approach for fabricating nano flake forest in polymer structures for various industrial applications.
\end{abstract}

Keywords: polymer composites, recycling, coatings, thin film, flake

\section{Introduction}

Inspired by biological materials, effort has been made to replicate similar technology. Active research on wettability property finds tremendous applications in key industries and in daily life, which is precisely controlled by geometrical pattern and chemical composition of the surface. The self-cleaning property of good water repellent surface with water contact angle of more than $150^{\circ}$ and angle of hysteresis less than $10^{\circ}$ has stimulated researchers to replicate multifunctional properties of natural materials for fabricating artificial superhydrophobic surfaces [1-4]. The water wettability is determined by water-material interaction and surface morphology [5]. Superhydrophobic surfaces have received great interest due to their multifunctional applications in different areas such as self-cleaning, anticorrosion, drag reduction, ice-mitigation, window glasses, antiicing textile, micro/macro fluid channels, optical devices, electronic and photonic material chemical sensors etc. [6-11]. Slippery surfaces are generally obtained by a combination of low surface energy materials and hierarchal structures, with trapped air pockets on which water droplets forms a liquid ball [12-17]. Earlier investigations on the surface texture of hydrophobicity were reported in terms of Wenzel and Cassie-Baxter model considering the water wettability of solid surface. Since then, low surface energy materials and fabrication techniques have been explored to design and create superhydrophobic surfaces $[18,19]$.

Nanostructured superhydrophobic surfaces have been fabricated using silica based materials and tridecafluoro-1,1,2,2-tetrahydrooctyldimethylchlorosilane (TFCS, $\left.\mathrm{CF}_{3}\left(\mathrm{CF}_{2}\right)_{5}\left(\mathrm{CH}_{2}\right)_{2}\left(\mathrm{CH}_{3}\right)_{2} \mathrm{SiCl}\right)$ through dip coating technique [20]. Shang et al. [21] have fabricated superhydrophobic silica film on glass substrates using polystyrene particles and DFMS (dodecafluoroheptyl-methyl-dimethoxysilane) as low surface energy material. Superhydrophobic sur-

\footnotetext{
${ }^{\text {*Corresponding author, e-mail: meetkbs@gmail.com }}$

(C) BME-PT
} 
faces using sodium chloride, sodium nitrate followed by fluorination treatment exhibiting water contact angle of $166^{\circ}[22,23]$. Each technique so far invented requires significant advancement and innovation in fabrication process and materials for real and exact industrial applications of superhydrophobic surfaces.

Considerable attention has been drawn on fabrication of hydrophobic surface using polymer/clay nanocomposite structures. Uniformly dispersed clay either as intercalated or delaminated results in nanocomposite formation however, non-dispersed clay shows no formation of nanocomposites. Enhancement of mechanical properties leads to the formation of exfoliated nanocomposite as compared to those observed for intercalated systems. It has been suggested that layered materials may be beneficial for the enhancement of polymer properties. Once clay materials are dispersed uniformly in a polymer matrix, the properties can be largely enhanced [24, 25]. Graphite as a layered structured material demonstrates enhancement of properties. It consists of carbon layers in an alternate stacking sequence with covalently bonded carbon atoms in a hexagonal arrangement within the layers. The typical d-spacing between the carbon layers in graphite is approximately $0.335 \mathrm{~nm}[24,25]$. Graphite and graphite powder are valued in industrial applications due to its lubricating properties. Based on these observations, it would be assumed that there should be improvement of properties observed for the graphitepolymer composites. Micron sized particles are indispensable materials for industries due to their unique size dependent properties (such as optical, mechanical, and electrical) largely differ from their bulk materials. Such particles have a different tendency for adhesion and aggregation, thus it is necessary to control their aggregation/dispersion phenomena in order to use them in functional materials and final products. However, controlling the stability of suspension in organic media is still a challenging issue [26-31].

WEP is extensively used as thermal insulation, due to its moisture resistance, lifetime durability and flame retardancy. It is the largest commodity polymer with total demand more than 80 million tonnes per annum. Most of the used WEP materials are not recycled effectively and hence pose serious disposal issues. However, the impact of WEP on the environment has drawn a great interest. The present work focuses on the study of wettability property of WEP which is used as binder and graphite powder as additive for investigating the hydrophobic properties. Literature reports the use of fluoropolymers or organic/inorganic materials to achieve highly hydrophobic surfaces. We have proposed an alternative cost-effective versatile method for achieving hydrophobic surfaces without fluorination treatment.

\section{Experimental}

\subsection{Materials}

Graphite powder (250 $\mu \mathrm{m}, 99.99 \%)$, toluene (anhydrous, 99.8\%), acetone (ACS reagent, $\geq 99.5 \%$ ), and ethanol (ACS reagent, $\geq 99.5 \%$ (200 proof), absolute) were purchased from Sigma-Aldrich (India) and used as received. Glass slides $(30 \times 35 \times 3 \mathrm{~mm})$ were received from Fisher Scientific (India). WEP having excellent properties such as thermal insulation, water resistance, compressive strength, non-abrasive to delicate parts are procured from POLYFOAM Corporation (USA) and used as received.

\subsection{Procedure for cleaning glass slides}

Glass slides were ultrasonicated (Sonicator ModelEI-6LH-SP, Sl. No- 1209-122, India) at $20 \mathrm{kHz}$ and $20 \mathrm{~W}$ in $20 \mathrm{~mL}$ ethanol for $15 \mathrm{~min}$ followed by ultrasonication with deionised water twice for next $5 \mathrm{~min}$ [32]. These cleaned glass slides were ready for use as substrates for hydrophobic coating.

\subsection{Preparation of homogeneous WEP composites}

In a typical process for fabrication of polystyrene blend, varying concentration of WEP (2-6 g) was mixed with $10 \mathrm{~mL}$ toluene. The viscosity of resulted solution was measured by Brookfield Viscometer. Appropriate amount of graphite powder (1-7 wt \%) was dispersed in WEP solution followed by ultrasonication at $40^{\circ} \mathrm{C}$. The viscosity of $2 \mathrm{~g}$ WEP in $10 \mathrm{~mL}$ toluene was observed to be $900 \mathrm{mPa} \cdot \mathrm{s}$ consistency. It is observed that viscosity of samples increases drastically with increase in concentration of WEP (3 to $6 \mathrm{~g}$ ). Since solvent evaporation is high during the spin coating process, higher viscosity of the solution results in non-uniform layer on the substrate. For achieving uniform thin layer on the glass slide, it is necessary to control the viscosity of the 
solution. It was observed that viscosity imparted by mixture of $2 \mathrm{~g}$ WEP in $10 \mathrm{~mL}$ toluene is suitable for formation of uniform thin layer on substrate.

\subsection{Fabrication of hydrophobic coating}

The optimized WEP/graphite solution was spin coated on a cleaned glass slide using spin coater, (Holmarc Model No. HO-TH-05, India). Briefly, two drops of solution were placed on a glass slide and spin coated at $2000 \mathrm{rpm}$ for $90 \mathrm{sec}$. This step is repeated thrice for fabrication of a dense layer on the surface of the substrate. The thickness of spin coated samples was optimized to 20 micron and was maintained for comparative study of wettability property. Figure 1 illustrates schematically the fabrication process for formation of hydrophobic coating on the glass substrates.

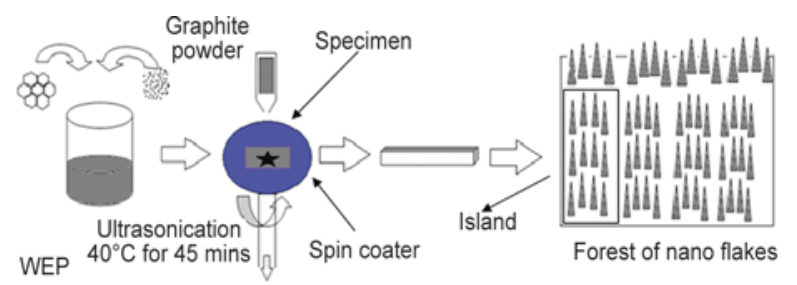

Figure 1. Schematic representation of the process for fabrication WEP film

\subsection{Films characterization}

The thickness of the coated layer was measured by 3D non contact surface profile $\left(\mathrm{NewView}^{\mathrm{TM}} 7100\right.$, Middlefield, USA). The morphology of the samples was observed using FE-SEM (JSM-6700F, JEOL, Japan). The roughness of the resulted coatings was evaluated using AFM (Asylum research, an Oxford Instrument company, UK) in tapping mode. The stability of the dispersed graphite powder in the polymer blend was monitored by the surface area analyzer (XiGo Nano Tools, Acorn Area, UK). This instrument was used for characterizing the stability of micro/nano sized particles present in the solution for fixed time duration. The stability of particles in the solution leads to homogeneous dispersion of particles i.e. particles do not aggregate or settle at the stipulated time period. The stability of the solvent (toluene), WEP/toluene, WEP/toluene/graphite was individually measured using the Acorn Area software version 0.82 . These measurements were carried out in NMR tubes having outer diameter $5 \mathrm{~mm} .0 .5 \mathrm{~mL}$ samples were placed in different NMR tubes such that the tubes are filled till the minimum height of $54 \mathrm{~mm}$ ensuring that no air bubbles are trapped. The tubes were capped to prevent drying of samples. For accurate results, the tubes were filled to the same volume. Initially, the bulk relaxation time $\left(T_{1}\right)$ of pure solvent (toluene) expressed in ms was quantified and saved as reference value for determining the relaxation time $\left(T_{2}\right)$ for polymer composite (EPF/graphite $4 \mathrm{wt} \%$, and $\mathrm{WEP} /$ graphite $5 \mathrm{wt} \%$ ). Surface wettability property of the samples was measured by the sessile drop technique [33]. The water contact angle (WCA) measurements were carried out on a Krüss DSA100 (Germany) contact angle goniometer with deionised water at an ambient temperature. During water contact angle measurement, $4 \mu \mathrm{L}$ of DI water was delivered from the syringe to form a sessile drop on the surface of the glass slide. An average of five measurements was used for the analysis.

\section{Results and discussion}

Surface modification of the particles is required to achieve enhanced stability of micron-sized particles in liquid media. This is done by introducing polymeric materials to generate an effective steric repulsive force from the polymer chains. This repulsive force increases the surface charge which controls the suspension rate of particles in the liquid medium. The nature of polymeric materials (hyrophilic/ hydrophobic) also plays a major role for generation of steric repulsive force for effective dispersion and stability of particles. For dispersion of hydrophobic powders such as $\mathrm{SiC}, \mathrm{CNT}$, Coal, etc., polymers with hydrophilic group or hydrophobic groups are often used as a surfactant. For hydrophobic surface modification of particles is a well-known technique for obtaining good dispersion and stability. The hydrophobic segments aids in adsorption of dispersant on to hydrophobic particles. Aromatic compounds such as styrene is used to make an effective adsorption of hydrophobic particles by means of hydrophobic and pi-pi interactions [34-37]. PEI (polyethyleneimine) is also used as hydrophobic segments, which is known to improve the stability of $\mathrm{SiC}$ and CNT in aqueous media by PEI [38]. Uhl and Wilkie [24] and Min et al. [25] have reported that parallel hexagonal networks of carbon atoms in graphite sheet are held together at certain spacing by weak van der Waals forces. During sonication process, $(20 \mathrm{kHz}$ and $20 \mathrm{~W}$ ) the individual layer of hexagonal network of carbon atoms in graphite particles are detached eas- 


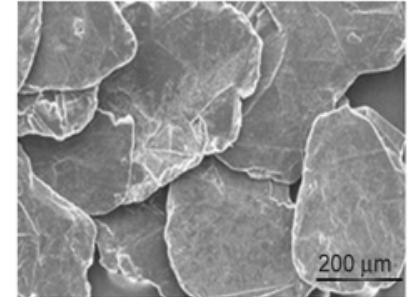

a)

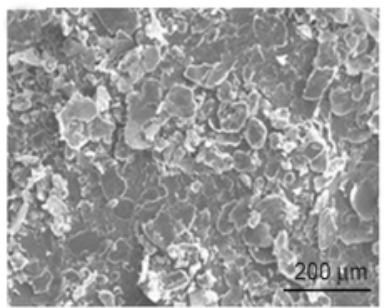

b)

Figure 2. (a) SEM of graphite as supplied, (b) SEM of ultrasonicated graphite

ily due to weak van der Waals forces which leads to reduction in particle size from 250 to less than $50 \mu \mathrm{m}$ which was confirmed in FESEM micrographs (Figure 2a-2b). Hence, sonication was contunued for $45 \mathrm{~min}$. However, in the present WEP/graphite composite no charge effect was observed. Toluene is a nonpolar solvent, there is no steric effect and charge formation. Aggregation of small sized graphite particles $(<50 \mu \mathrm{m})$ occurs in the solution, which has strong impact on the morphology of the films. These flakes are deposited during spin coating process and forms islands of flakes. During sonication homogeneous dispersion occurs, which has stability up to $30 \mathrm{~min}$. Aggregation occurs during spin coating which results in formation of islands of flakes. These islands enhance the surface roughness which increases the hydrophobicity surface. The quality of dispersion of particles in the NMR tubes affects the suspension of graphite particles in the composite. 4 and $5 \mathrm{wt} \%$ graphite in WEP blend showed good dispersion of particles for $30 \mathrm{~min}$. However, suspension of graphite particles from $\mathrm{WEP} / 4 \mathrm{wt} \%$ graphite was found to be better as compared to suspension of graphite particles in $\mathrm{WEP} / 5 \mathrm{wt} \%$ graphite. This is due to settling of graphite from $\mathrm{WEP} / 5 \mathrm{wt} \%$ graphite, which leads to variation in the relaxation time as shown in Figure 3.

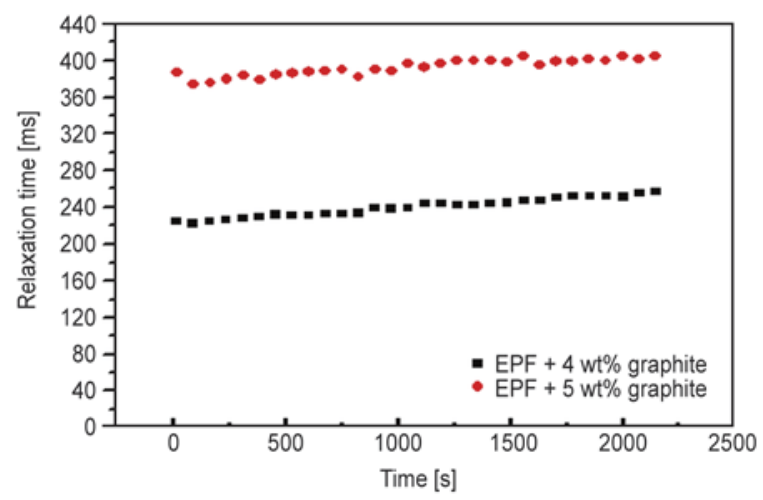

Figure 3. Stability test of graphite powders of $4 \mathrm{wt} \%$ and $5 \mathrm{wt} \%$ in the polymer solution
Uniform relaxation time was observed for $\mathrm{WEP} / 4 \mathrm{wt} \%$ graphite confirming no aggregation or settling of particles. Thus the relaxation time required for settling of particles was measured through surface area analyzer and used to study the stability of particles in solution. These experimental results facilitate correlation of surface morphology of polymer film with wettability property.

Polymer films with uniform thickness of $20 \mu \mathrm{m}$ thin film were fabricated using spin coater were fabricated using varied concentration of additives. The effect of concentration of additives on the water contact angle as shown in Figure 4 presents stepwise loss of hydrophilic property with increase in weight percentage of graphite powder. With $1 \mathrm{wt} \%$ graphite in the blend, WCA enhances from 89 to $98^{\circ}$. Further increase in graphite concentration to $2 \mathrm{wt} \%$, WCA shows marginally increase in WCA from 98 to $101^{\circ}$. With 3 and $5 \mathrm{wt} \%$, WCA was obtained to be 104 and $119^{\circ}$ respectively which shows better hydrophobicity. Decrease in WCA was observed with further increase in graphite content. WCA in however increased from 119 to $129^{\circ}$ with an increase in thickness of the film from 20 to $30 \mu \mathrm{m}$ on the glass slide. This increase in hydrophobicity of film correlates the surface morphology of the developed film with different concentration of graphite loading.

The wetting behaviour of the surface depends upon the surface chemistry and surface topology. The surface topology follows the Wenzel and CassieBaxter water contact model, in which surface roughness increases the apparent water contact angle. For hydrophilic surfaces, the interaction between the substrate surface and water is favoured but for hydrophobic surfaces, this interaction is prevented due to the presence of surface roughness resulting in spontaneous increase in water contact angle. Systemati-

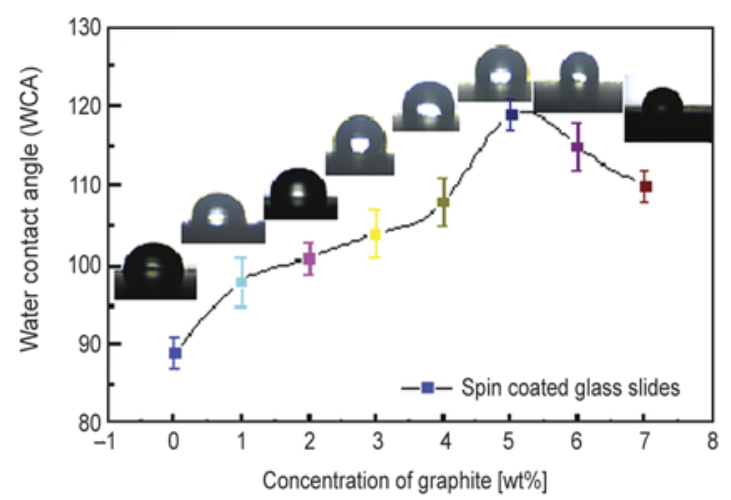

Figure 4. Effect of the different concentration of graphite on water contact angle 
cally study the surface morphology of blend films were studied by formation of layer by layer of stable surface of graphite with WEP was generated on the surface of etched glass by spin coating technique. Surface roughness is a measure of the texture of the surface. It is quantified by the vertical deviation of the real surface from its ideal surface. The double layer structure improves the surface roughness as compared to single layer structure. When second layer structure is introduced over the single layer structure, the deposition of graphite particles takes place over the top first layer, which enhances roughness factor. This phenomenon is based on Wenzel Equation (1) [18]:

$\cos \left(\theta^{\mathrm{w}}\right)=r \cos \theta$

where $\theta$ is the maximum WCA observed, $r$ is the theoretical roughness calculated and $\theta^{\mathrm{w}}$ is Wenzel water contact angle. The arithmetic mean height of surface along $Z$ axis, of double layer structure is more than the single layer structure and hence hydrophobicity increases. The aggregation of graphite flakes occurs over each layer and arithmetic mean height of surface along $Z$ axis is enhanced. The mean height affects the roughness $(r)$ of the surface and hence hydrophobicity is enhanced. Table 1 presents gradual increase in WCA with increase in $r$ values indicating WCA is related to air fraction on the surface.

FESEM studies of the polymer films revealed the evolution of different morphological images as shown in Figure 5. By spin coating, the solution of intrinsically hydrophobic polystyrene smooth film is formed on the surface of glass substrate. Previous studies regarding multiscale structures especially micro-nano scale structures, which are advanta-

Table 1. Water contact angle and roughness of the surface of different polymer blends

\begin{tabular}{|c|c|c|c|}
\hline $\begin{array}{c}\text { Concentration } \\
\text { of graphite } \\
{[\mathbf{w t} \% \mathbf{]}}\end{array}$ & $\begin{array}{c}\text { Average } \\
\text { roughness, Sa } \\
{[\mathbf{n m}]}\end{array}$ & $\begin{array}{c}\text { Average } \\
\text { height of peak } \\
{[\mathbf{n m}]}\end{array}$ & $\begin{array}{c}\text { Water contact } \\
\text { angle } \\
{\left[{ }^{\circ}\right]}\end{array}$ \\
\hline 0 & 20.009 & 14 & 89 \\
\hline 1 & 80.141 & 30 & 98 \\
\hline 2 & 86.418 & 40 & 101 \\
\hline 3 & 88.064 & 65 & 104 \\
\hline 4 & 93.567 & 79 & 108 \\
\hline 5 & 109.078 & 110 & 119 \\
\hline 6 & 104.560 & 102 & 115 \\
\hline 7 & 95.120 & 80 & 110 \\
\hline
\end{tabular}




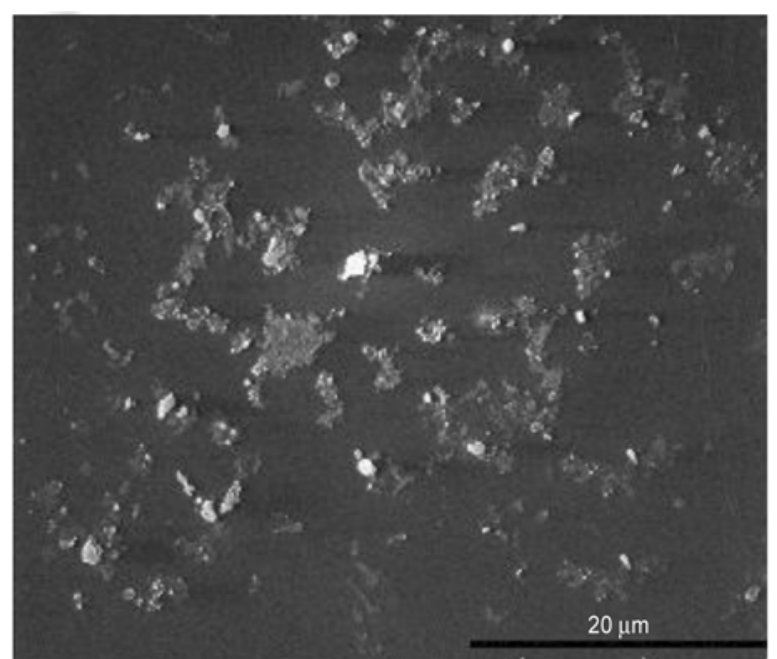

a)

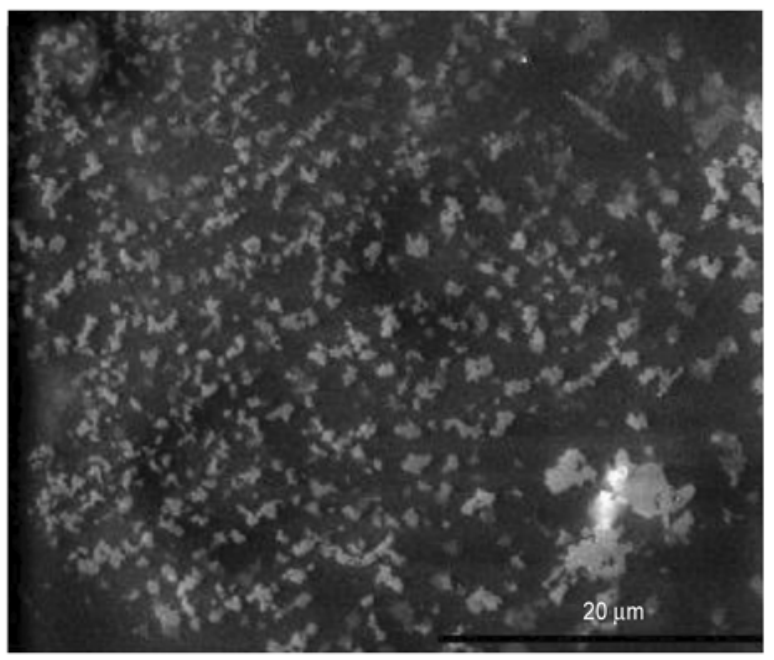

c)

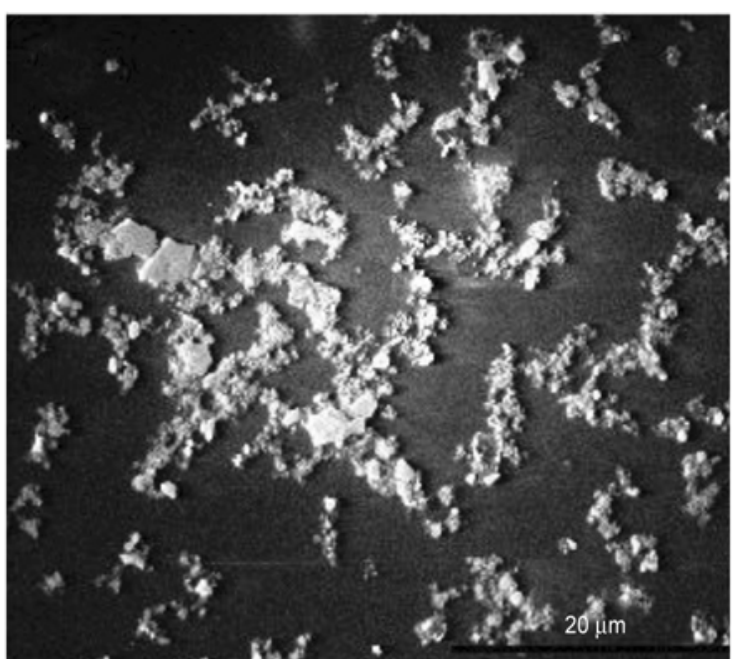

b)

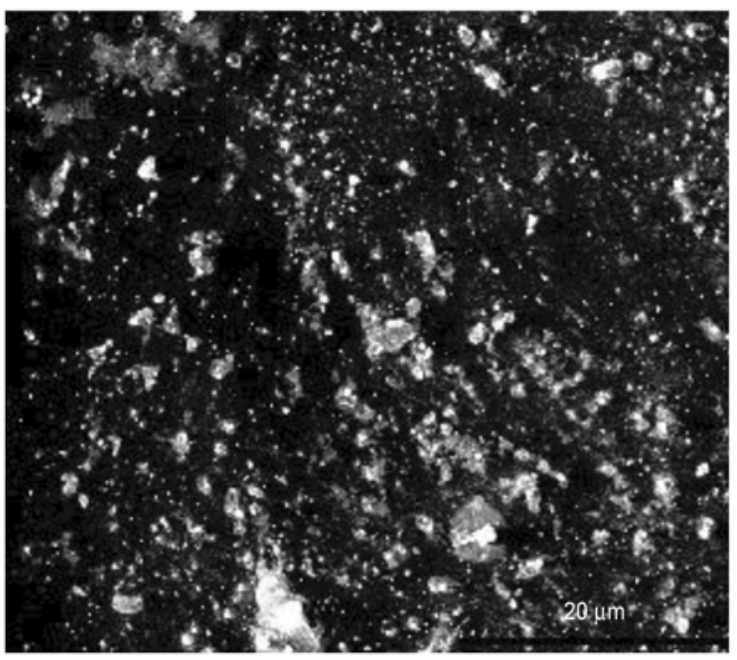

d)

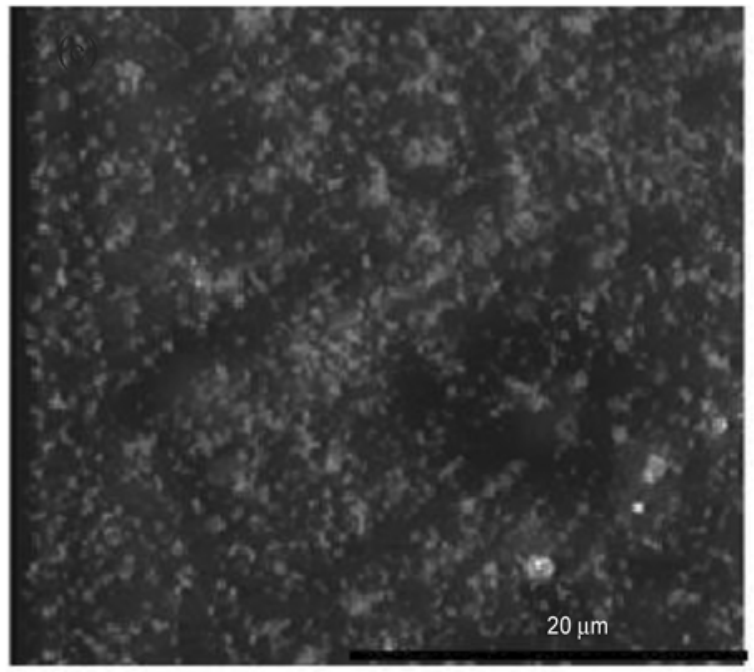

e)

Figure 5. FE-SEM images of the WEP blend with different concentration of graphite powder (a) $1 \mathrm{wt} \%$; (b) $2 \mathrm{wt} \%$; (c) $3 \mathrm{wt} \%$; and (d) $4 \mathrm{wt} \%$; (e) $5 \mathrm{wt} \%$

formed on the surface with hierarchal structures. Surface roughness was evaluated by the vertical deviation of a real surface from its original form. The average roughness of polymer film, $\mathrm{Sa}=$
80.141 was quantified for WEP/1 $\mathrm{wt} \%$ graphite film. Introduction of more number of islands with increase in graphite content to $2 \mathrm{wt} \%$ increased the average roughness to 86.418 . Further increase in 


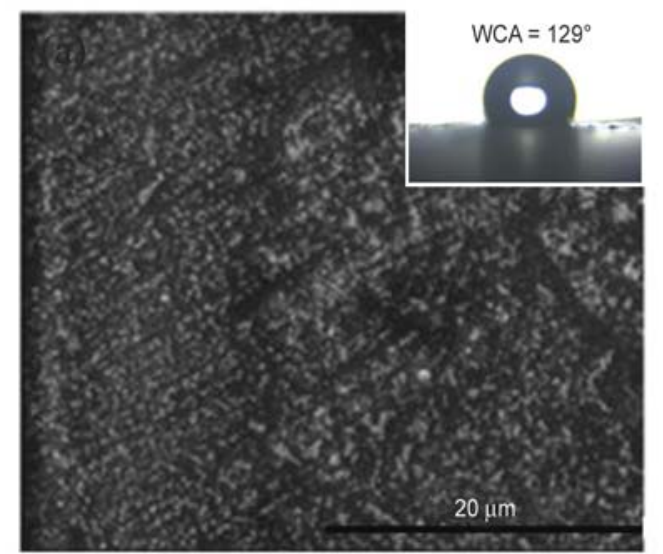

a)

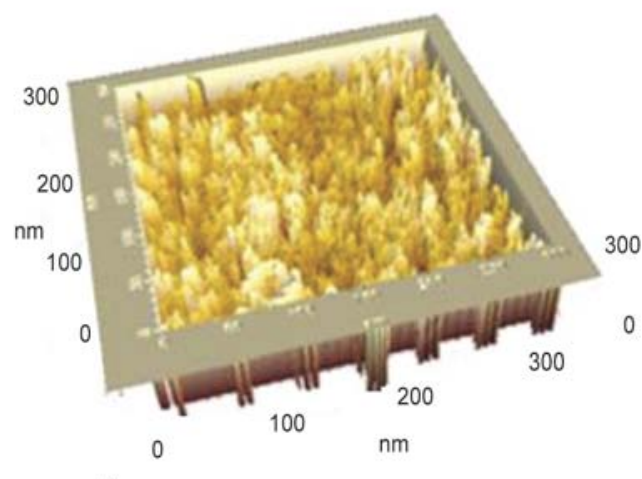

b)

Figure 6. (a) FE-SEM images of the WEP blend (four layers) with $5 \mathrm{wt} \%$ of graphite powder, (b) AFM image WEP film with $5 \mathrm{wt} \%$ graphite powder for four layers

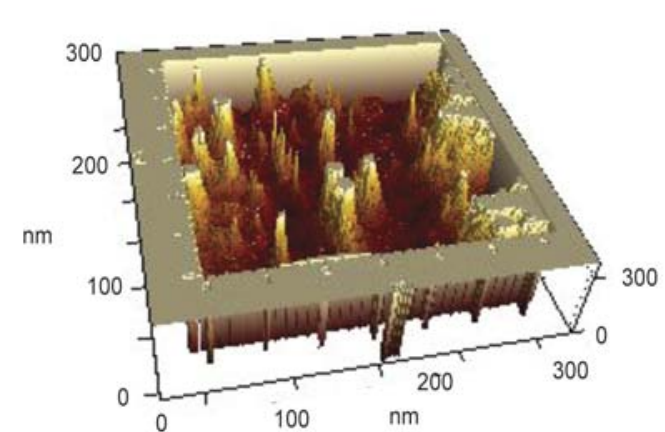

a)

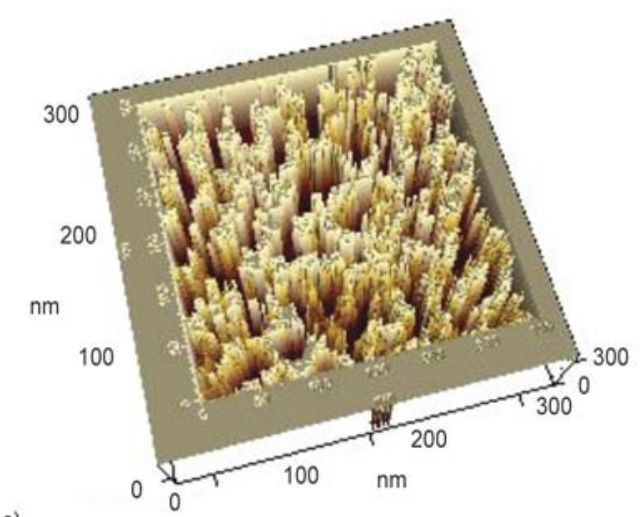

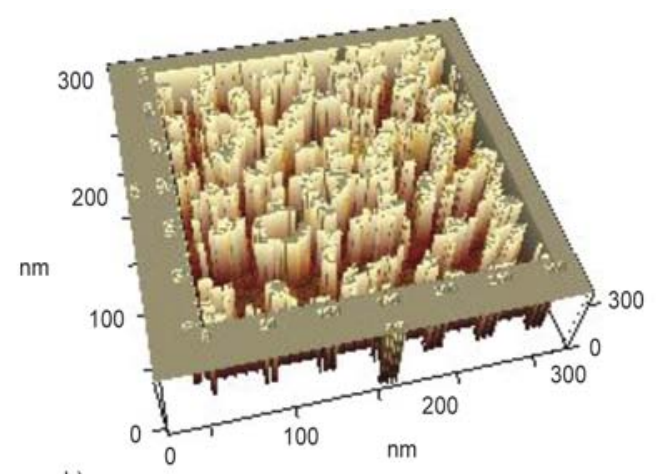

b)

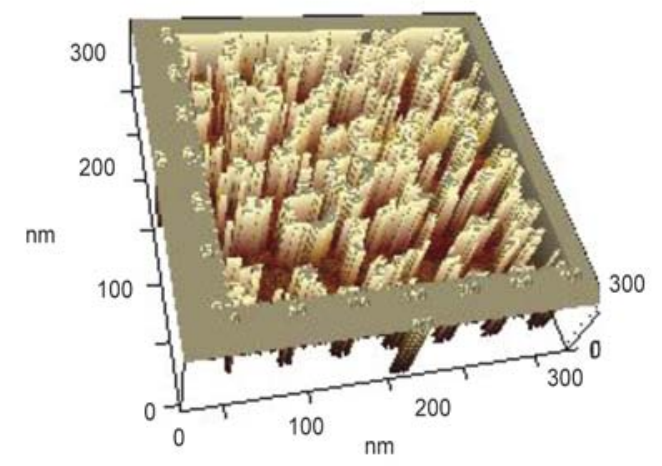

d)

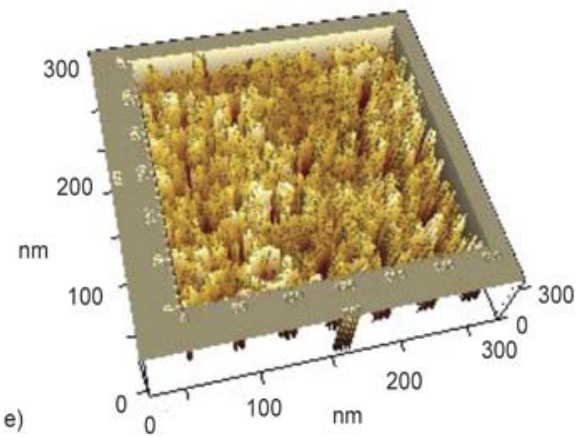

Figure 7. Tapping mode of AFM topology and 2D images of WEP film with content of graphite powder of (a) 1 wt $\%$; (b) $2 \mathrm{wt} \%$; (c) $3 \mathrm{wt} \%$; (d) $4 \mathrm{wt} \%$; (e) $5 \mathrm{wt} \%$ 
graphite content to $3 \mathrm{wt} \%$, shows increase in number of nano flakes formation which combines to form the forest as shown in Figure 7c. Our observation correlates well the roughness of the film with the water contact angle. Similarly, the relation between surface roughness and water contact angle for other samples was revealed [45]. The surface roughness and water contact angle data collected from all the samples are shown in Table 1. Maximum surface roughness of 109.078 was quantified for graphite concentration of $5 \mathrm{wt} \%$. This increase in roughness was attributed to the formation of a large number of islands. We also noticed that maximum height of a single flake $(160 \mathrm{~nm})$ and separation between two flakes $(0.2 \mathrm{~nm})$ on the surface enables the anisotropic slippery surface. A single island consists of a forest of nano flakes formed on the surface, which covers maximum area with increasing graphite content. This trend translated into the increased hydrophobicity was indicated by WCAs (Figure 4). With increased graphite concentration the distance between the nano flakes decreased gradually. The average height of nano flakes also decreases simultaneously and hence roughness loosened widely. But with increasing thickness of the polymer film layer, surface roughness improves, which was clearly observed from in Figure 6b [46]. In Wenzel model, water droplet floats on the projection surface. The average height of the peaks is experimentally calculated as $110 \mathrm{~nm}$ for graphite concentration of $5 \mathrm{wt} \%$ from AFM images. With the introduction of more concentration of graphite powders, more nano flakes are formed on the surface, which reduces the average height of the peak. It was observed that with $7 \mathrm{wt} \%$ graphite, the average height of peak reduces to $80 \mathrm{~nm}$. Similar observations are also revealed with the deposition of four layers on the surface. This observation supports the idea that surface topology plays an important role determining the wettability behaviour of the surface than surface composition.

\section{Conclusions}

In this study, we have described a facile and cost effective technique for fabrication of hydrophobic surface with good water repellent property using WEP, which can be self assembled into a forest of nano flakes by the spin coating process. These forests of nano flakes are combined to form small islands on the surface. The network of nano flakes of the spin coated sample was effectively controlled by the concentration of graphite, and the wettability property was analyzed by measurement of water contact angle. The stability of dispersed graphite powders with WEP materials clearly shows excellent suspension behaviour in the liquid medium. The induced roughnesses in the samples seem to be correlated to the surface morphology. The proposed water contact angle values were reflected from the surface topology. WCA was enhanced up to $129^{\circ}$ due to modification of surface film by increase in thickness of film. This method could provide an alternative approach for fabricating superhydrophobic materials with hierarchical structures. We hope that this approach could provide to prepare the superhydrophobic surface with low adhesion.

\section{Acknowledgements}

Authors greatly appreciate the financial support from the Defence Institute of Advanced Technology, Pune. The authors would like to thank the Vice Chancellor Dr Prahlada, DIAT, Naval Head Quarters and 'DIAT-NANO project EPIPR/ ER/1003883/M/01/908/2012/D (R\&D)/ 1416' for encouragement and support. We are grateful to Dr. P. G. Ghosal, DMRL and Dr S. Datar for characterisation support.

\section{References}

[1] Barthlott W., Neinhuis C.: Purity of the sacred lotus, or escape from contamination in biological surfaces. Planta, 202, 1-8 (1997).

DOI: $10.1007 / \mathrm{s} 004250050096$

[2] Gao X., Jiang L.: Water-repellent legs of water striders. Nature, 232, 36-38 (2004). DOI: $10.1038 / 432036 \mathrm{a}$

[3] Zhai L., Berg M. C., Cebeci F. Ç., Kim Y., Milwid J. M., Rubner M. F., Cohen R. E.: Patterned superhydrophobic surfaces: Toward a synthetic mimic of the Namib desert beetle. Nano Letter, 6, 1213-1217 (2006). DOI: $10.1021 / \mathrm{n} 1060644 \mathrm{q}$

[4] Goswami D., Medda S. K., De G.: Superhydrophobic films on glass surface derived from trimethylsilanized silica gel nanoparticles. ACS Applied Materials Interfaces, 3, 3440-3447 (2011). DOI: $10.1021 / \mathrm{am} 200666 \mathrm{~m}$

[5] Bravo-Gutiérrez M. E., Castro M., Hernández-Machado A., Poiré C. E.: Controlling viscoelastic flow in microchannels with slip. Langmuir, 27, 2075-2079 (2011). DOI: $10.1021 / 1 \mathrm{l} 103520 \mathrm{a}$

[6] Blossey R.: Self-cleaning surfaces - Virtual realities. Nature Materials, 2, 301-306 (2003).

DOI: $10.1038 /$ NMAT856 
[7] Choi C. H., Kim C. J.: Large slip of aqueous liquid flow over a nanoengineered superhydrophobic surface. Physical Review Letters, 96, 066001/1-066001/4 (2006).

DOI: 10.1103/PhysRevLett.96.066001

[8] Choi C-H., Ulmanella U., Kim J., Ho C-M., Kim C-J.: Effective slip and friction reduction in nanograted superhydrophobic microchannels. Physics of Fluids, 18, 087105/1-087105/8 (2006).

DOI: $10.1063 / 1.2337669$

[9] Zhang F., Zhao L., Chen H., Xu S., Evans D. G., Duan $\mathrm{X}$.: Corrosion resistance of superhydrophobic layered double hydroxide films on aluminum. Angewandte Chemie International Edition, 47, 2466-2469 (2008). DOI: $10.1002 /$ anie. 200704694

[10] Cao L., Jones A. K., Sikka V. K., Wu J., Gao D.: Antiicing superhydrophobic coatings. Langmuir, 25, 12444 12448 (2009).

DOI: $10.1021 / 1 \mathrm{a902882b}$

[11] Lee H. J.: Design and development of anti-icing textile surfaces. Journal of Materials Science, 47, 5114-5120 (2012) DOI: $10.1007 / \mathrm{s} 10853-012-6386-2$

[12] Sarkar D. K., Farzaneh M.: Superhydrophobic coatings with reduced ice adhesion. Journal Adhesion Science and Technology, 23, 1215-1237 (2009).

DOI: $10.1163 / 156856109 X 433964$

[13] Saleema N., Farzaneh M., Paynter R. W., Sarkar D. K.: Prevention of ice accretion on aluminum surfaces by enhancing their hydrophobic properties. Journal of Adhesion Science and Technology, 25, 27-40 (2011). DOI: $10.1163 / 016942410 X 508064$

[14] Saleema N., Farzaneh M.: Thermal effect on superhydrophobic performance of stearic acid modified $\mathrm{ZnO}$ nanotowers. Applied Surface Science, 254, 2690-2695 (2008).

DOI: 10.1016/j.apsusc.2007.10.004

[15] Sarkar D. K., Farzaneh M., Paynter R. W.: Superhydrophobic properties of ultrathin rf-sputtered Teflon films coated etched aluminum surfaces. Materials Letters, 62, 1226-1229 (2008).

DOI: $10.1016 /$ j.matlet.2007.08.051

[16] Sarkar D. K., Farzaneh M., Paynter R.: Wetting and superhydrophobic properties of PECVD grown hydrocarbon and fluorinated-hydrocarbon coatings. Applied Surface Science, 256, 3698-3701 (2010).

DOI: $10.1016 /$ j.apsusc.2009.12.049

[17] Safaee A., Sarkar D. K., Farzaneh M.: Superhydrophobic properties of silver-coated films on copper surface by galvanic exchange reaction. Applied Surface Science, 254, 2493-2498 (2008)

DOI: $10.1016 /$ j.apsusc. 2007.09.073

[18] Wenzel R. N.: Resistance of solid surfaces to wetting by water. Industrial and Engineering Chemistry, 28, 988-994 (1936). DOI: $10.1021 / \mathrm{ie} 50320 \mathrm{a} 024$
[19] Cassie A. B. D., Baxter S.: Wettability of porous surfaces. Transactions of the Faraday Society, 40, 546551 (1944).

DOI: $10.1039 /$ TF9444000546

[20] Shang H. M., Wang Y., Takahashi K., Cao G. Z., Li D., Xia Y. N.: Nanostructured superhydrophobic surfaces. Journal of Material Science, 40, 3587-3591 (2005) DOI: $10.1007 / \mathrm{s} 10853-005-2892-9$

[21] Shang Q., Gao L., Liu H., Xiao G.: Fabrication of superhydrophobic silica film by removing polystyrene spheres. Journal of Sol-Gel Science Technology, 59, 334-337 (2011).

DOI: $10.1007 / \mathrm{s} 10971-011-2507-7$

[22] Song J., Xu W., Lu Y.: One-step electrochemical machining of superhydrophobic surfaces on aluminum substrates. Journal of Materials Science, 47, 162-168 (2012).

DOI: $10.1007 / \mathrm{s} 10853-011-5783-2$

[23] Xu W., Song J., Sun J., Dou Q., Fan X.: Fabrication of superhydrophobic surfaces on aluminum substrates using $\mathrm{NaNO}_{3}$ electrolytes. Journal of Materials Science, 46, 5925-5930 (2011). DOI: $10.1007 / \mathrm{s} 10853-011-5546-0$

[24] Uhl F. M., Wilkie C. A: Polystyrene/graphite nanocomposites: Effect on thermal stability. Polymer Degradation and Stability, 76, 111-122 (2002). DOI: $10.1016 / \mathrm{S} 0141-3910(02) 00003-4$

[25] Min X., Sun L., Liu J., Li Y., Gong K.: Synthesis and properties of polystyrene/graphite nanocomposites. Polymer, 43, 2245-2248 (2002).

DOI: $10.1016 / \mathrm{S} 0032-3861(02) 00022-8$

[26] Iijima M., Kamiya H.: Surface modification for improving the stability of nanoparticles in liquid media. KONA Powder and Particle Journal, 27, 119-129 (2009).

[27] Marignier J. L., Belloni J., Delcourt M. O., Chevalier J. P.: Microaggregates of non-noble metals and bimetallic alloys prepared by radiation-induced reduction. Nature, 317, 344-345 (1985). DOI: $10.1038 / 317344 \mathrm{a} 0$

[28] Alivisatos P.: Semiconductor clusters, nanocrystals, and quantum dots. Science, 271, 933-937 (1996). DOI: $10.1126 /$ science.271.5251.933

[29] Ozin G. A.: Nanochemistry: Synthesis in diminishing dimensions. Advanced Materials, 4, 612-649 (1992). DOI: 10.1002/adma.19920041003

[30] Jun Y-W., Huh Y-M., Choi J-S., Lee J-H., Song H-T., Kim S., Yoon S., Kim K-S., Shin J-S., Suh J-S., Cheon J.: Nanoscale size effect of magnetic nanocrystals and their utilization for cancer diagnosis via magnetic resonance imaging. Journal of American Chemical Society, 127, 5732-5733 (2005). DOI: $10.1021 /$ ja0422155

[31] Dick K., Dhanasekaran T., Zhang Z., Meisel D.: Sizedependent melting of silica-encapsulated gold nanoparticles. Journal of American Chemical Society, 124, 2312-2317 (2002). DOI: $10.1021 / \mathrm{ja} 017281 \mathrm{a}$ 
[32] Yin L., Wang Y., Ding J., Wang Q., Chen Q.: Water condensation on superhydrophobic aluminum surfaces with different low-surface-energy coatings. Applied Surface Science, 258, 4063-4068 (2012).

DOI: $10.1016 /$ j.apsusc. 2011.12.100

[33] Chen P., Hu Y., Wei C.: Preparation of superhydrophilic mesoporous $\mathrm{SiO}_{2}$ thin films. Applied Surface Science, 258, 4334-4338 (2012).

DOI: $10.1016 /$ j.apsusc.2011.12.109

[34] Spyriouni T., Economou I. G., Theodorou D. N.: Molecular simulation of $\alpha$-olefins using a new united-atom potential model: Vapor-liquid equilibria of pure compounds and mixtures. Journal of American Ceramic Society, 121, 3407-3413 (1999). DOI: $10.1021 / \mathrm{ja} 982453 \mathrm{y}$

[35] Xue C-H., Shi M-M., Yan Q-X., Shao Z., Gao Y., Wu G., Zhang X-B., Yang Y., Chen H-Z., Wang M.: Preparation of water-soluble multi-walled carbon nanotubes by polymer dispersant assisted exfoliation. Nanotechnology, 19, 115605-115612 (2008).

DOI: $10.1088 / 0957-4484 / 19 / 11 / 115605$

[36] Kakui T., Kamiya H.: Effect of sodium aromatic sulfonate group in anionic polymer dispersant on the viscosity of coal-water mixtures. Energy and Fuels, 18, 652-658 (2004).

DOI: $10.1021 / \mathrm{ef030154a}$

[37] Delozier D. M., Watson K. A., Smith J. G., Clancy T. C., Connell J. W.: Investigation of aromatic/aliphatic polyimides as dispersants for single wall carbon nanotubes. Macromolecules, 39, 1731-1739 (2006).

DOI: $10.1021 / \mathrm{ma} 051826 \mathrm{u}$

[38] Zhang T., Zhang Z., Dong M., Zhang J., Lin Q., Jiang D.: The influence of polyethylene imine on the gelcasting of sic with two different initiators. Journal of American Ceramic Society, 90, 3748-3756 (2007). DOI: $\underline{10.1111 / \mathrm{j} .1551-2916.2007 .01992 . \mathrm{x}}$
[39] Karmouch R., Guy G. R.: Superhydrophobic wind turbine blade surfaces obtained by a simple deposition of silica nanoparticles embedded in epoxy. Applied Surface Science, 257, 665-669 (2010). DOI: $10.1016 /$ j.apsusc. 2010.07.041

[40] He Z., Ma M., Xu X., Wang J., Chen F., Deng H., Wang K., Zhang Q., Fu Q.: Fabrication of superhydrophobic coating via a facile and versatile method based on nanoparticle aggregates. Applied Surface Science, 258, 2544-2550 (2012).

DOI: $10.1016 /$ j.apsusc. 2011.10 .090

[41] Xu M., Lu N., Qi D., Xu H., Wang Y., Shi S., Chi L.: Fabrication of superhydrophobic polymer films with hierarchical silver microbowl array structures. Journal of Colloid and Interface Science, 360, 300-304 (2011). DOI: 10.1016/j.jcis.2011.04.048

[42] Wang J., Chen X., Kang Y., Yang G., Yu L., Zhang P.: Preparation of superhydrophobic poly(methyl methacrylate)-silicon dioxide nanocomposite films. Applied Surface Science, 257, 1473-1477 (2010).

DOI: $10.1016 / j$.apsusc.2010.08.075

[43] Manoudis P., Papadopoulou S., Karapanagiotis I., Tsakalof A., Zuburtikudis I., Panayiotou C.: Polymersilica nanoparticles composite films as protective coatings for stone-based monuments. Journal of Physics: Conference Series, 61, 1361-1365 (2007). DOI: $10.1088 / 1742-6596 / 61 / 1 / 269$

[44] Ramaratnam K., Iyer S. K., Kinnan M. K., Chumanov G., Brown P. J., Luzinov I.: Ultrahydrophobic textiles using nanoparticles: Lotus approach. Journal of Engineered Fibers, 3, 1-14 (2008).

[45] Athauda T. J., Decker D. S., Ozer R. R.: Effect of surface metrology on the wettability of $\mathrm{SiO}_{2}$ nanoparticle coating. Materials Letters, 67, 338-341 (2012). DOI: 10.1016/j.matlet.2011.09.100

[46] Meng L-Y., Park S-J.: Effect of fluorination of carbon nanotubes on superhydrophobic properties of fluorobased films. Journal of Colloid and Interface Science, 342, 559-563 (2010). DOI: $10.1016 /$ j.jcis.2009.10.022 